University of Louisville

ThinkIR: The University of Louisville's Institutional Repository

Faculty Scholarship

$1-4-2017$

\title{
Effect of trehalose as an additive to dimethyl sulfoxide solutions on ice formation, cellular viability, and metabolism.
}

\author{
Jason Solocinski \\ University of Michigan - Dearborn \\ Quinn Osgood \\ University of Michigan - Dearborn \\ Mian Wang \\ University of Michigan - Dearborn \\ Aaron Connolly \\ University of Michigan - Dearborn \\ Michael A. Menze \\ University of Louisville \\ See next page for additional authors
}

Follow this and additional works at: https://ir.library.louisville.edu/faculty

Part of the Biology Commons, and the Cell and Developmental Biology Commons

Original Publication Information

Solocinski, Jason, et al. "Effect of Trehalose as an Additive to Dimethyl Sulfoxide Solutions on Ice Formation, Cellular Viability, and Metabolism." 2017. Cryobiology https://doi.org/10.1016/

j.cryobiol.2017.01.001

This Article is brought to you for free and open access by ThinkIR: The University of Louisville's Institutional Repository. It has been accepted for inclusion in Faculty Scholarship by an authorized administrator of ThinkIR: The University of Louisville's Institutional Repository. For more information, please contact thinkir@louisville.edu. 


\section{Authors}

Jason Solocinski, Quinn Osgood, Mian Wang, Aaron Connolly, Michael A. Menze, and Nilay Chakraborty

This article is available at ThinkIR: The University of Louisville's Institutional Repository: https://ir.library.louisville.edu/ 
1 Effect of Trehalose as an Additive to Dimethyl Sulfoxide Solutions on

2 Ice Formation, Cellular Viability, and Metabolism

4 Jason Solocinski ${ }^{1}$, Quinn Osgood ${ }^{1}$, Mian Wang ${ }^{1,3}$, Aaron Connolly ${ }^{1}$, Michael A. Menze ${ }^{2}$,

$5 \quad$ Nilay Chakraborty ${ }^{1}$

6

$7 \quad{ }^{1}$ Department of Mechanical Engineering, University of Michigan-Dearborn

84901 Evergreen Road, Dearborn MI 48128, Unites States

9 2Department of Biology, University of Louisville, Louisville, KY 40292, Unites States

$10{ }^{3}$ Current Address: Department of Mechanical Engineering, University of Minnesota,

11 Minneapolis, MN 55455, Unites States

12 Address for reprint requests and other correspondence: Nilay Chakraborty and Michael

13 A. Menze Tel.: 313-583-6788, Fax: 313-593-3851, Email: nilay@umich.edu; Tel.: (502)

14 852-8962, Fax: 502- 852-0725, Email: michael.menze@louisville.edu

15

16

17 Keywords: Hepatocellular Carcinoma Cells, Cryopreservation, Raman

18 Microspectroscopy, Respiration, Osmotic Stress, Mitochondria 


\section{Abstract}

Cryopreservation is the only established method for long-term preservation of cells

22 and cellular material. This technique involves preservation of cells and cellular

23 components in the presence of cryoprotective agents (CPAs) at liquid nitrogen

24 temperatures $\left(-196^{\circ} \mathrm{C}\right)$. The organic solvent dimethyl sulfoxide (Me2SO) is one of the most

25 commonly utilized CPAs and has been used with various levels of success depending on

26 the type of cells. In recent years, to improve cryogenic outcomes, the non-reducing

27 disaccharide trehalose has been used as an additive to $\mathrm{Me}_{2} \mathrm{SO}-$ based freezing solutions.

28 Trehalose is a naturally occurring non-toxic compound found in bacteria, fungi, plants,

29 and invertebrates which has been shown to provide cellular protection during water-

30 limited states. The mechanism by which trehalose improves cryopreservation outcomes

31 remains not fully understood. Raman microspectroscopy is a powerful tool to provide

32 valuable insight into the nature of interactions among water, trehalose, and $\mathrm{Me}_{2} \mathrm{SO}$ during

33 cryopreservation. We found that the addition of trehalose to $\mathrm{Me}_{2} \mathrm{SO}$ based CPA solutions

34 dramatically reduces the area per ice crystals while increasing the number of ice crystals

35 formed when cooled to -40 or $-80{ }^{\circ} \mathrm{C}$. Differences in ice-formation patterns were found to

36 have a direct impact on cellular viability. Despite the osmotic stress caused by addition of

$37100 \mathrm{mM}$ trehalose, improvement in cellular viability was observed. However, the

38 substantial increase in osmotic pressure caused by trehalose concentrations above

$39100 \mathrm{mM}$ may offset the beneficial effects of changing the morphology of the ice crystals

40 achieved by addition of this sugar. 


\section{Introduction}

Cryoprotective agents (CPAs), are traditionally used to ensure survival of cellular samples at cryogenic temperatures. Due to toxicity concerns of penetrating CPAs such as dimethyl sulfoxide ( $\mathrm{Me}_{2} \mathrm{SO}$ ), several additives such as glycerol [1], disaccharides (e.g. trehalose, sucrose $[1 ; 2 ; 3])$, amino acids (e.g. proline $[2 ; 4 ; 5]$ ), and proteins (e.g. sericin [6]) have been used in recent years. Several organisms in nature are frequently exposed to subzero temperatures and a common strategy in these organisms is to accumulate biocompatible osmolytes such as trehalose before the onset of water loss due to freezing, drying, or both $[7 ; 8 ; 9]$. Trehalose has been found to improve the cryogenic outcome in a variety of biological materials including mammalian cells and cellular monolayers $[4 ; 5$; $10 ; 11 ; 12]$. However, the actual mechanism for improvement of cellular viability in presence of trehalose following cryopreservation remains poorly understood [13; 14]. Here, we present an in-depth analysis of the effect of trehalose addition to a $\mathrm{Me}_{2} \mathrm{SO}-$ based freezing solution on ice-formation, cumulative osmotic stress, viability, and postthaw metabolic activity of human hepatocellular carcinoma (HepG2) cells.

At low cooling rates $\left(\sim 1^{\circ} \mathrm{C} / \mathrm{min}\right)$ 'solution effects' injury stemming from exposure of cells to a hypertonic extracellular environment for extended period of time is the primary cause of cellular damage $[15 ; 16 ; 17 ; 18]$. During freezing, water crystalizes in the extracellular environment - a process that increases the solute concentration in the nonfrozen water fraction surrounding the cells. In addition to osmotic stress, extracellular ice morphology can have a strong bearing on cellular viability [19]. However, most experimental techniques do not allow characterizing spatial differences in ice morphology and solute distribution in frozen systems. Spatially correlated Raman microspectroscopy 
64 techniques were used at -40 and $-80^{\circ} \mathrm{C}$ to characterize changes in the ice formation and solute distribution after addition of trehalose to $\mathrm{Me}_{2} \mathrm{SO}$ based freezing solutions.

Vibrational Raman microspectroscopy is a highly sensitive technique that relies on

67 detection of vibration in molecular moieties when excited with laser irradiation [20; 21]. Since the vibrational information is specific to the chemical bonds and symmetry of molecules, Raman microspectroscopy provides a fingerprint by which a molecule can be identified $[21 ; 22]$. This extends to different physical states in the same molecule such as

71 the transition from water to ice [23; 24]. Therefore, it is feasible to use Raman

72 microspectroscopy to study cryoprotective formulations and investigate the distribution,

73 state, and concentration of compounds at different sub-zero temperatures. While $\mathrm{Me}_{2} \mathrm{SO}$

74 has been widely used as a penetrating cryoprotectants and is known to depress the

75 freezing point of aqueous solutions [25; 26], at relatively low concentration (<1M) $\mathrm{Me}_{2} \mathrm{SO}$

76 has little influence on the average water-water hydrogen bonding strength [27]. In contrast

77 to $\mathrm{Me}_{2} \mathrm{SO}$, Raman microspectroscopic observations [28] and molecular dynamic 78 simulations [29; 30] have revealed that trehalose promotes a destructive effect on the 79 tetrahedral hydrogen-bond network of pure water [30]. These studies suggest that in 80 presence of trehalose, water binds stronger to the sugar than to other water molecules. 81 Trehalose obstructs the water-crystallization process, thereby destroying the water 82 network and forming a sugar-water network [31]. At low temperatures formation of ice 83 creates a partially dehydrated environment and while the additive trehalose may be 84 excluded from the immediate vicinity of the biomolecules of interest [32] in presence of 85 the sugar, ice formation occurs at lower temperatures but at more independent nucleation 86 sites [33]. 
We hypothesized that the destructuring effect of trehalose on water-water

88 hydrogen bonding will be maintained in presence of $\mathrm{Me}_{2} \mathrm{SO}$. Therefore, by reducing the

89 availability of water molecules to join a tetrahedral hydrogen network that plays a

90 formative role in creating ice crystals during freezing, an overall smaller ice crystal size

91 may be observed in presence of water, trehalose, and $\mathrm{M}_{2} \mathrm{SO}$ compared to the binary

92 water $\mathrm{Me}_{2} \mathrm{SO}$ system. In this study a highly sensitive confocal Raman microspectroscopic

93 (CRM) system was used to generate spatially correlated chemical maps of the distribution

94 of ice, $\mathrm{Me}_{2} \mathrm{SO}$, and trehalose in the frozen systems. Special attention was paid to the

95 effect of trehalose concentration on the formation and distribution of ice crystals and the 96 recovery of metabolic functions after cryopreservation of HepG2 cells. 


\section{Materials and Methods}

99

100

101

102

103

104

105

106

107

108

109

110

111

112

113

114

115

116

117 Samples were cooled to -40 and $-80{ }^{\circ} \mathrm{C}$ at a rate of $1^{\circ} \mathrm{C} / \mathrm{min}$, and then held for 118

119

\subsection{Sample preparation}

Low endotoxin $\alpha$, $\alpha$-trehalose dihydrate was obtained from Pfanstiehl Inc. (Waukegan, IL) and dimethyl sulfoxide (Me2SO) was procured from Sigma Aldrich (St. Louis, MO). Solutions of $10 \%(\mathrm{v} / \mathrm{v}) \mathrm{Me} 2 \mathrm{SO}$ were made by mixing $10 \%$ pure $\mathrm{Me}_{2} \mathrm{SO}$ with 90\% phosphate buffered saline (PBS) solution from Sigma Aldrich (St. Louis, MO) volume by volume and then dissolving trehalose to reach final concentrations of $0 \mathrm{mM}, 100 \mathrm{mM}$, and $300 \mathrm{mM}$ trehalose. These solutions were used in the confocal Raman microspectroscopy studies and in the cell freezing studies.

\subsection{Low temperature confocal Raman microspectroscopy}

Low temperature Raman measurements were conducted using a customized confocal microscope and Raman spectrometer combination (UHTS 300, WITec Instruments Crop, Germany). Raman spectra were collected using a highly sensitive EMCCD camera (Andor Technology, UK). A 532nm solid-state laser was used for excitation and images were captured using a 10X objective (Carl Zeiss, Germany). A liquid nitrogen cooled freezing stage (FDCS 196, Linkam Scientific Instrument, UK) was integrated with the microscopy setup and was used to cool the samples at a predetermined rate. For each experiment, the freezing stage with $20 \mu$ l solution of sample was mounted on the Raman microscope stage with a custom-made stage adaptor. approximately 10 minutes at each temperature before the spectral information was collected. Spatially correlated hyperspectral Raman images were created using the 
120 Raman signals collected from a window of $50 \times 50 \mu \mathrm{m}$. Each array of Raman scans was

121 collected using a low integration time $(0.3 \mathrm{~s})$ to minimize impact of laser irradiation on the

122 ice crystals formed. Each experiment was repeated 3 times and the confocal Raman

123 images presented here are representative for all 3 repetitions.

$124 \quad 2.3$ Image Processing

125 Images were processed using the open source software Image $\mathrm{J}$ [34]. All images were 126 processed for identification and quantification of ice crystals using a standard bandpass 127 filter for particle analysis. A threshold was applied to convert raw hyperspectral images to 128 a binary image. The Watershed segmentation algorithm [35] in Image $\mathrm{J}$ was used to 129 prevent the individual ice crystals from merging to one another and the Particle Analysis 130 tool was used to quantify both the number and area of the ice crystals.

1312.4 Cell culture and cryopreservation Human hepatocellular carcinoma (HepG2) cells were obtained from the American 133 Type Culture Collection (Manassas, VA), and grown in $75 \mathrm{~cm}^{2}$ cell culture flasks (Corning 134 Incorporated, Corning, NY). Standard culture medium for HepG2 cells was composed of 135 Opti-MEM I (Gibco, Carlsbad, CA) supplemented with 10\% fetal bovine serum (FBS) 136 (Gibco) and penicillin-streptomycin solution to yield final concentrations of 100 units $/ \mathrm{mL}$ 137 penicillin $\mathrm{G}$ and $100 \mu \mathrm{g} / \mathrm{mL}$ streptomycin sulfate (HyClone-Thermo Scientific, Logan, UT). 138 Cells were cultured at $37^{\circ} \mathrm{C}$ in a humidified atmosphere of $5 \% \mathrm{CO}_{2}$ and $95 \%$ air. Upon 139 reaching $80-90 \%$ confluency, cells were dissociated using $0.25 \%$ trypsin plus $1 \mathrm{mM}$ EDTA 140 in a balanced salt solution for 10 min, and trypsin activity was stopped by adding fully 141 supplemented medium to the flask followed by centrifugation for $5 \mathrm{~min}$ at $200 \mathrm{x} \mathrm{g}$. The 
142 cells were washed once with fully supplemented medium and the final cell pellet was

143 resuspended in one of the three different solutions containing CPAs previously

144 mentioned. Cell samples were diluted in the CPA solutions to a concentration of $1 \times 10^{6}$

145 cells $/ \mathrm{mL}$. A volume of $1 \mathrm{~mL}$ of the samples was transferred into type $\mathrm{D}$ micro tubes

146 (Sarstedt, Radnor, PA), and placed into a passive freezing device (Cool Cell LX,

147 Biocision, Menlo Park, CA), which provides a cooling rate of $1^{\circ} \mathrm{C} / \mathrm{min}$. After loading with

148 samples, the freezing device was quickly transferred to a

$149-80^{\circ} \mathrm{C}$ commercial freezer for $24 \mathrm{~h}$. The following day, the tubes were quickly collected

150 and transferred to a LN2 storage container.

1512.5 Mathematical modeling of cumulative osmotic stress

152

153

154

155

156

157

158

The progressive loss of osmotically active intracellular water with the increase of extracellular osmolality during freezing at $1^{\circ} \mathrm{C} / \mathrm{min}$ was modeled based on the formulation as discussed by Fahy, 1981. The cumulative osmotic stress experienced by the cell was defined as loss of osmotically active water volume over time. The differential decrease in osmotically active volume of intracellular water with change of temperature was indicated by following equation (Fahy, 1981):

$$
\square \square=\frac{-\square_{\square} \square^{\square\left(\square-\square_{\square}\right)} \square \square(\square+273.15) \square \square}{\square \bar{\square}_{\square}} \times \ln \left[\frac{1-\left(\frac{\square}{\left(\square / \square_{\square} \square_{\square}+1\right)}\right)-\left(\frac{\square}{\left(\square / \square \square \square_{\square}+1\right)^{2}}\right)}{1+0.00966 \square+4.1025 \times 10^{-5} \square^{2}}\right]
$$

Here $A$ is the total surface area of the cell, $\square_{\square}$ is the hydraulic conductivity of the cell membrane at a given temperature $\square_{\square}, R$ is the universal gas constant, $B$ is the cooling rate, $b$ is the temperature coefficient of the hydraulic conductivity, $\bar{\square}_{\square}$ is the partial molar volume of the water, $\mathrm{V}$ is the volume of intracellular water, and $\square_{\square}$ is the number of moles 
163 of solute in the cells. Relevant parametric values for HepG2 cells are listed in Table 1. S 164 and I are parameters that are dependent on the non-aqueous mole fraction of the 165 constituents of the freezing medium. The following parametric relationships were used for 166 calculating $S$ and I for each of the CPA formulations with trehalose.

$$
\square=3.55 \square_{\square}+1.8 \text { and } \square=0.076 \square_{\square}+0.86 \text { where } \square_{\square}=\square_{\square} /\left(\square_{\square}+2 \square_{\square}+\square_{\square}\right)
$$

168 Here, $\square_{\square}$ is the number of moles of $\mathrm{Me}_{2} \mathrm{SO}, \square_{\square}$ is the number of moles of salts in the 169 freezing solution and finally, $\square_{\square}$ is the number of moles of trehalose in the freezing 170 solution. The calculated values for the parameters $S$ and I are presented in Table 2 for 171 each of the CPA formulations. A computer code written in Mathematica 8 (Wolfram

172 Research, Champaign, IL) was used to solve the set of equations described above.

1732.6 Post-thaw viability and metabolic profile analysis

Following storage over LN2 for a day, individual microtubes were collected and 175 quickly warmed to physiological temperature using a water bath maintained at $37^{\circ} \mathrm{C}$. In 176 order to remove the CPAs in the solution, cells were collected using centrifugation 177 followed by resuspension in standard culture medium. Cells were enumerated with a 178 Bright Line ${ }^{\mathrm{TM}}$ hemocytometer (Hausser Scientific, Horsham, PA) and membrane integrity 179 was assessed using trypan blue exclusion assay. The oxygen consumption rates (OCRs) 180 of HepG2 cells cryopreserved under different CPA conditions were measured using the 181 XFp Extracellular Flux Analyzer (Seahorse Biosciences, North Billerica, MA) on days 1 182 and 3 post thawing. The Seahorse XFp analyzer operates by creating a transient chamber 183 so that cellular oxygen consumption rates can be monitored. Post thaw cell samples were 184 plated on XFp plates at $4 \times 10^{4}$ cells per well and incubated for $24 \mathrm{~h}$ before respiration rates 
185 were measured. Preceding experimentation, the XFp cartridges (Seahorse Bioscience, 186 North Billerica, MA) were hydrated with XFp calibrant (Seahorse Bioscience) and stored 187 at $37^{\circ} \mathrm{C}$ for $24 \mathrm{~h}$. One-hour prior measuring cellular respiration, the cell culture media was 188 aspirated from the individual culture wells and a medium containing DMEM (Dulbecco's 189 Modified Eagle's medium, Seahorse Bioscience) plus 2mM L-Glutamine and 20mM 190 glucose (Sigma Aldrich, St. Louis, MO) was added. The plate was maintained for $1 \mathrm{~h}$ at $19137^{\circ} \mathrm{C}$ and ambient atmosphere. The XFp cartridge was loaded with a suite of reagents 192 yielding the following final concentrations in the cell sample: oligomycin $(1 \mu \mathrm{M})$, carbonyl-

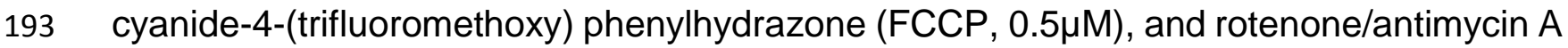
$194(0.5 \mu \mathrm{M})$. Oligomycin acts as $\mathrm{F}_{0} \mathrm{~F}_{1}$-ATPase inhibitor and oxygen consumption rates 195 measured in presence of this inhibitor indicate mitochondrial leak respiration, while FCCP 196 acts as an uncoupling agent which collapses the mitochondrial proton gradient and 197 thereby uncouples the oxidation system from the phosphorylation system, maximizing 198 oxygen consumption rates. To estimate the contribution of non-mitochondrial processes

199 to overall oxygen flux, rotenone and antimycin A were added to inhibit complex I and III 200 of the respiratory system.

\section{$201 \quad 2.7$ Statistical analysis}




\section{Results}

207

\subsection{Confocal Raman microscopy (CRM)}

Spatially correlated CRM can be used for simultaneous identification and localization of multiple molecular moieties by analyzing individual chemical signatures. In

210 Fig. 1, a typical Raman spectrum of our tertiary ice, trehalose, and Me2SO system is used 211 to spatially correlate the distribution of compounds in a $50 \times 50 \mu \mathrm{m}^{2}$ sample window at $21240^{\circ} \mathrm{C}$. The hyperspectral images were extracted using the appropriate characteristic 213 wavelengths for each of the compounds and brightness correlates with increased 214 compound concentration. It is interesting to note that channels of high $\mathrm{Me}_{2} \mathrm{SO}$ 215 concentrations were found to be embedded between ice crystals while trehalose seems 216 to be more ubiquitously distributed throughout both the ice and $\mathrm{Me}_{2} \mathrm{SO}$ rich regions of the 217 sample.

In this study, primary emphasis was laid on investigating the ice-formation 219 characteristics at different trehalose concentrations with decreasing temperature. As shown in Fig. 2, ice crystals (bright area) were surrounded by narrow channels (dark

221 area), which are rich in $\mathrm{Me}_{2} \mathrm{SO}$ due to the presence of the solutes rejected by the 222 nucleating and growing ice phase. Average ice crystal area was determined and a clear 223 trend in formation of ice crystals having smaller surface areas with increase in trehalose 224 concentration and decrease in temperature was observed. At $-40^{\circ} \mathrm{C}$ in samples containing $22510 \% \mathrm{Me}_{2} \mathrm{SO}$ alone relatively large ice crystals $\left(\mathrm{A}_{\mathrm{avg}}=138.2 \mu \mathrm{m}^{2}\right)$ with sharp and angular 226 boundaries developed. Upon addition of $100 \mathrm{mM}$ trehalose to $10 \% \mathrm{Me}_{2} \mathrm{SO}$, ice crystals 227 were smaller $\left(A_{a v e}=114.9 \mu \mathrm{m}^{2}\right)$ than in presence of $10 \% \mathrm{Me}_{2} \mathrm{SO}$ alone. Furthermore, the 228 ice crystals displayed more rounded and smoother boundaries. Further increases in 
229 trehalose concentration to $300 \mathrm{mM}$, caused additional decreases in ice crystal area $\left(A_{\text {ave }}=\right.$

$23054.3 \mu \mathrm{m}^{2}$ ) and boundaries appear to be more rounded than under the two other conditions

231 investigated. Upon cooling to $-80^{\circ} \mathrm{C}$, all the samples exhibit additional decreases in

232 average ice crystal size compared to $-40^{\circ} \mathrm{C}$. At $-80^{\circ} \mathrm{C}$ the ice crystals had a relatively

233 uniform distribution, and the same decreasing trend of ice crystal area with increasing

234 trehalose concentrations found for samples at $-40^{\circ} \mathrm{C}$, was observed. It is noteworthy that

235 for $10 \% \mathrm{Me}_{2} \mathrm{SO}$ plus $300 \mathrm{mM}$ trehalose at $-80^{\circ} \mathrm{C}$, the structure of ice crystals is very

236 different compared to all other conditions and the ice crystals were extremely small ( $A_{a v e}=$

$23719.8 \mu \mathrm{m}^{2}$ ) forming a more network-like structure.

Based on the morphology and number of ice crystals in the hyperspectral images

239 the average size distribution and frequency of crystals per unit of viewing area was

240 calculated. As shown in Fig. 3A, ice crystal area decreases while the number of ice

241 crystals per unit viewing area increases with increasing trehalose concentration. This

242 effect was observed at both temperatures, but the increase in number of ice crystals with

243 increase in trehalose concentration was most pronounced at $-80^{\circ} \mathrm{C}$ (Fig. 3B). While a

244 similar trend in crystal formation was observed in absence of $\mathrm{Me}_{2} \mathrm{SO}$ and in presence of

245 only trehalose the crystal sizes are significantly bigger (Supplementary Figures I and II),

246 indicating that addition of $\mathrm{Me}_{2} \mathrm{SO}$ does in fact play an important role in determining and

247 reducing the size of the ice crystals formed.

Ice formation in the extracellular environment increases the solute concentration

249 in the unfrozen section around the cells. While $\mathrm{Me}_{2} \mathrm{SO}$ is a penetrating cryoprotectant, 250 trehalose remains predominantly outside the cell contributing to the increasing 251 extracellular solute concentration [26; 36]. These freezing events outside the cellular 
252 environment have a direct impact on the osmotically active cell volume as cells maintain

253 an osmotic equilibrium with the extracellular environment [37]. Guided by the hydraulic

254 conductivity of the cell membrane and the rate of decrease in temperature, cells become

255 partially dehydrated due to osmotically active water leaving the cytoplasm and organelles

256 [38]. At low cooling rates, such dehydration can lead to osmotic stress mediated injury in

257 cells commonly known as 'solution effects' injury [16; 39], and can be considered as the

258 predominant injury mechanism at low cooling rates [40]. Such injury can be

259 mathematically modeled as the cumulative effect of volumetric reduction of cells owing to

260 the loss of water [40]. Fig. 4 describes the relationship between temperature and

261 osmotically active cell volume. As expected, at a freezing rate of $1^{\circ} \mathrm{C} / \mathrm{min}$, we see that

262 cumulative osmotic stress increases substantially starting at $-15^{\circ} \mathrm{C}$ when frozen in a $10 \%$

$263 \mathrm{Me}_{2} \mathrm{SO}$ solution. Addition of trehalose to $10 \% \mathrm{Me}_{2} \mathrm{SO}$ solution increases the cumulative

264 osmotic stress experienced by cells (Fig. 5). As expected, we see that upon addition of

$265300 \mathrm{mM}$ trehalose cells experience increased reduction of osmotically active water

266 volume leading to higher cumulative osmotic stresses (Fig. 5). According to the model

267 developed here, most of the injuries occur in -5 to $-20^{\circ} \mathrm{C}$ range where there is a substantial

268 difference in percent increase of cumulative osmotic stress when $300 \mathrm{mM}$ trehalose is

269 added to $\mathrm{Me}_{2} \mathrm{SO}$.

$270 \quad 3.2$ Cell growth and metabolic profile analysis

271 Membrane integrity after freeze thawing and growth of HepG2 cells was measured

272 in order to assess the physiological consequences of trehalose addition to the CPA

273 solution. Despite the increase in cumulative osmotic stress in presence of $100 \mathrm{mM}$

274 trehalose compared to $\mathrm{Me}_{2} \mathrm{SO}$ alone, membrane integrity was significantly higher for cells 
275 frozen in presence of $100 \mathrm{mM}$ trehalose compared to $0 \mathrm{mM}$ trehalose (Fig. 6A). 276 Furthermore, no significant differences in growth behavior were found between cells 277 frozen without trehalose or in presence of $100 \mathrm{mM}$ of the sugar (Fig. 6B). After an initial 278 lagging phase of about 3 days, cell numbers increased rapidly over the next 2-3 days, 279 followed by reduced proliferation rates due to contact inhibition. However, due to the 280 substantial higher cumulative osmotic stress experienced at $300 \mathrm{mM}$ trehalose, cells 281 frozen in this CPA showed both lower membrane integrity and longer delayed growth 282 performance compared to the $100 \mathrm{mM}$ trehalose samples (Fig. 6A, B). Oxygen 283 consumption rates (OCR) provided an additional appraisal of cellular functions of cells 284 after cryopreservation. In agreement with growth performance, analysis of the basal OCR 285 data for each CPA condition on days 1 and 3 post-thawing showed increases in cellular 286 respiration over time for each CPA employed (Fig. 7A). Increases in OCR were followed 287 by increases in oligomycin inhibited and FCCP uncoupled respiration rates (Fig. 7B, C). 288 Furthermore, a slight increase in the background oxygen flux after addition of rotenone 289 and antimycin-a was also observed (Fig. 7D). In summary, no substantial differences in 290 bioenergetic parameters were observed for cells frozen in the three different CPAs after 2913 days of cell recovery. 


\section{Discussion}

294 The rate of freezing is a critical factor that determines the nature and extent of cellular 295 injury during cryoprocessing. At slow freezing rates $\left(1-10^{\circ} \mathrm{C} / \mathrm{min}\right)$ physical damage by 296 advancing ice crystals and prolonged exposure to hyperosmotic conditions are the 297 dominant injury mechanisms. On the other hand, at fast freezing rates $\left(>10^{\circ} \mathrm{C} / \mathrm{min}\right)$ 298 intracellular water fails to equilibrate with the rapidly increasing extracellular osmolality 299 due to physical limits associated with the hydraulic conductivity of the cell membrane and 300 the chances of formation of highly lethal intracellular ice increases $[41 ; 42]$.

Irrespective of the specific freezing rates, CPAs offer protective mechanisms to 302 prevent cellular injury during freezing. CPAs capable of permeating the cell membrane 303 (i.e. $\mathrm{Me}_{2} \mathrm{SO}$ ) play a role in preventing intracellular ice formation and are thought to 304 contribute to a vitrified environment in the intracellular space, whereas non-penetrating 305 cryoprotectants (i.e. polyethelene glycol) modulate the extracellular ice formation 306 characteristics [43] and are known to have osmolytic properties preventing membrane damage caused by hyperosmotic conditions in the extracellular environment during freezing [16]. One of the most significant drawbacks associated with use the of CPAs is 309 the fact that many CPAs, including the widely used compound Me2SO, are known to have significant cytotoxic effects both in short term and long term. Me $2 \mathrm{SO}$ has been reported

311 to cause translocation of apoptosis-inducing factors from mitochondria to nucleus and 312 poly-(ADP-ribose)-polymerase (PARP) activation [44]. Additionally, Me $2 \mathrm{SO}$ is reported to 313 induce pore formation in plasma membrane [45]. The toxicity associated with CPAs has 314 been a limiting step for the use of high CPA concentrations, and poses a significant 315 problem for application of cryopreservation protocols to a wide variety of cells including 
stem cells. At higher concentrations of $\mathrm{Me}_{2} \mathrm{SO}$, Molecular Dynamics (MD) simulations predict cell membrane loosening, pore formation, and eventual bilayer collapse [46].

One strategy to mitigate the risk of using toxic CPAs is to add cosolutes with cytoprotective properties as additives to the CPA formulation. While many additives have been used as cryoprotectants, it has been demonstrated that a majority of them fail to protect proteins and phospholipid bilayers from denaturation during dehydration stress experienced by cells during cryoprocessing [47]. It is interesting to note that disaccharides such as sucrose and trehalose are an exception and possess the ability to prevent protein denaturation and membrane fusion during cryoprocessing $[1 ; 48]$. Trehalose have been widely used as additives to cryoprotectants formulations in recent years [2; $3 ; 49 ; 50]$. Trehalose is a non-reducing disaccharide and it has been linked to extreme dehydration and low temperature tolerance in several cryptobiotic organisms [51]. Addition of 0.2$0.6 \mathrm{M}$ trehalose to a CPA containing $10 \% \mathrm{Me}_{2} \mathrm{SO}$ has been demonstrated to increase both post-thaw cell viability and plating efficiency in several mammalian cell types including primary human hepatocytes [52], human embryonic cells [10], and pancreatic islets [53]. However, the exact mechanism(s) by which trehalose protects cellular structures during cryopreservation remains unclear.

Due to the lack of dedicated trehalose transporter in mammalian cells, addition of trehalose to CPA formulations results in presence of trehalose predominantly in the extracellular space. The water replacement hypothesis [54] suggests that the hydroxyl groups of trehalose can substitute for the hydrogen bonding of water [5]. During cryopreservation as water molecules are being progressively removed from extracellular environment due to ice formation, trehalose may play a critical role in maintaining the 
339 integrity of the phospholipid structure of the cell membrane. In a comparable system 340 involving water loss, such as drying, it has been demonstrated that the loss of the 341 hydration effect of water is compensated by the presence of trehalose, thus preserving 342 the phospholipid bilayer. However, without trehalose, it has been found that desiccation 343 leads to heterogeneities in phospholipid packing and reduced acyl chain density, thereby 344 destabilizing the membrane and resulting in damage upon the influx of water [55]. As an 345 additive to CPA formulations, trehalose may play a similar role in minimizing cellular injury 346 during the partial dehydration created during cryoprocessing owing to the progressive ice 347 formation in extracellular space [56]. However, it needs to be noted that to offer maximal 348 protection during desiccation, trehalose has to be present on both sides of the plasma 349 membrane [14], which is not the case without a sugar loading strategy.

By employing Raman microspectroscopy we found that in absence of sugar 351 loading trehalose may exert a protective effect by modulating the nature of the 352 extracellular ice crystal formed (Fig. 1). Trehalose has been known to inhibit ice crystal 353 growth [33], and recent studies underscore a strong correlation between the ice-crystal 354 size and cell lethality [57]. Rapid growth of large ice crystals in extracellular medium 355 increases the possibility of damage to the cell membrane from advancing ice crystals. In 356 this study we demonstrate that presence of trehalose as an additive to the CPA 357 formulation can significantly influence the morphology, shape, and size of ice crystal 358 formation (Fig. 2) - which can in turn have a significant role to mitigate physical damage 359 due to advancing ice crystals during freezing. While similar studies have been reported 360 using optical microscopy [58], field emission electron microscopy systems including 361 cryoscanning electron microscopy (SEM), or transmission electron microscopy (TEM) 
362 [33], only the hyperspectral imaging technique using spatially correlated Raman 363 microspectroscopy system combines digital imaging and molecular/elemental 364 spectroscopy for material analysis. This technique provides the significant advantage of 365 spectroscopically identifying the true nature of ice formation pattern. The ice crystal 366 formation is indicated by appearance of a distinct peak in the symmetric spectral region 367 of the $\mathrm{OH}$ stretching peak $~ 3130 \mathrm{~cm}^{-1}$ wavenumbers [5; 59].

At low temperatures both the reduction in the free water, indicated by decreasing 369 intensity of the asymmetric portion of the $\mathrm{OH}$ stretching spectra (centered $\sim 3435 \mathrm{~cm}^{-1}$ ), 370 and enhancement of nucleation sites for ice crystals have been theorized to limit the 371 number of ice crystals in presence of trehalose. Hyperspectral images of the ice crystals 372 formed at both $-40^{\circ} \mathrm{C}$ and $-80^{\circ} \mathrm{C}$ both support this theory. The number of nucleation sites 373 indicated by the number of ice crystals increase significantly when $300 \mathrm{mM}$ trehalose in 374 added to the CPA solution. As temperature of the system is lowered at $1{ }^{\circ} \mathrm{C} / \mathrm{min}$ to $-80^{\circ} \mathrm{C}$, 375 this effect is significantly enhanced in comparison to the number of ice crystals formed at $376-40^{\circ} \mathrm{C}$. Systematic quantification of the ice crystal number and area as seen on the 377 hyperspectral images indicates that the number of ice crystals increases rapidly, and the 378 area per ice crystal decreases in turn (Figs. 3A, B). Surprisingly, while cumulative osmotic 379 stress increases with increasing trehalose concentrations (Figs. 4,5) membrane integrity 380 and metabolic activity is not negatively impacted (Figs. 6,7). These findings reinforce the 381 notion that ice crystal structure has direct impacts on cryopreservation outcomes and can 382 be modulated by the addition of sugar additives to the CPA solution. Experiments with 383 mesenchymal stem cell cells that are more sensitive to osmotic stress than HepG2 cells 384 are currently underway and should provide additional insights into the complex 
385 relationship between osmotic stress, ice-crystal morphology, and viability post thawing in 386 cryopreservation. 
387 Conflict of interest

The authors certify that they have no affiliations with or involvement in any 389 organization or entity with any financial interest (such as honoraria; educational grants; 390 participation in speakers' bureaus; membership, employment, consultancies, stock 391 ownership, or other equity interest; and expert testimony or patent-licensing 392 arrangements), or non-financial interest (such as personal or professional relationships) 393 in the subject matter or materials discussed in this manuscript.

\section{Statement of funding}

395 This research was funded by NSF grant numbers SusChEM 1510072 and CHE 1609440 396 to N.C. and IOS-1659970 to M.A.M. UM Research grant having project numbers U038727, 397 U051381, and U046888 are also acknowledged.

\section{Acknowledgments}

399 The authors would also like to acknowledge Tavis Ezell's help in procuring the Raman 400 instrumentation from WITec Instruments, GmbH. 
Fig. 1. A solution of $10 \% \mathrm{Me} 2 \mathrm{SO}$ and $100 \mathrm{mM}$ trehalose was frozen to $-40^{\circ} \mathrm{C}$ at $1^{\circ} \mathrm{C} / \mathrm{min}$.

404 Upon reaching stable crystal morphologies, individual hyperspectral images indicating 405 spatial concentrations of ice, $\mathrm{Me}_{2} \mathrm{SO}$, and trehalose were extracted by integrating 406 representative characteristic Raman peaks. The characteristic Raman peaks for each of 407 the components are labeled in the average spectrum of the scanned area. (Ice: $3130 \mathrm{~cm}^{-}$ 408 1, Me $2 \mathrm{SO}: 1426 \mathrm{~cm}^{-1}$, trehalose: $\left.855 \mathrm{~cm}^{-1}\right)$. Maximum intensities for each integrated peak 409 relative to zero were 685.3, 108.2, and 22.9 CCD cts for ice, $\mathrm{Me}_{2} \mathrm{SO}$, and trehalose, 410 respectively.

411 Fig. 2. Confocal Raman hyperspectral images of $10 \% \mathrm{Me}_{2} \mathrm{SO}$ solution in presence of $4120 \mathrm{mM}, 100 \mathrm{mM}$ and $300 \mathrm{mM}$ trehalose at $-40^{\circ} \mathrm{C}$ and $-80^{\circ} \mathrm{C}$. All the images are generated 413 by integrating the ice peak $\left(3130 \mathrm{~cm}^{-1}\right)$ from the corresponding Raman spectra.

414 Fig. 3. Numerical representation of crystal morphological properties extracted from 415 Raman hyperspectral images. Images were taken at $-40^{\circ} \mathrm{C}$ and $-80^{\circ} \mathrm{C}$ for each of the 416 three CPA solutions containing $10 \% \mathrm{Me}_{2} \mathrm{SO}$ solution with trehalose additives $(0 \mathrm{mM}$, 417 100mM, and $300 \mathrm{mM})$. Hyperspectral images were analyzed with ImageJ to extract 418 average number of ice crystals $(A)$ and average area per ice crystals $(B)$ were found via 419 ImageJ per sample window $(n=3)$.

420 Fig. 4. Normalized cell volume and cumulative osmotic stress at a cooling rate of $1^{\circ} \mathrm{C} / \mathrm{min}$. 421 Mathematica modeled parametric curves showing interaction of osmotically active cell 422 volume and cumulative osmotic stress from $0^{\circ} \mathrm{C}$ to $-80^{\circ} \mathrm{C}$. The osmotically active volume 423 of the cell is approximately $30 \%$ of the total cell volume. 
424 Fig. 5. Percent increase in cumulative osmotic stress with decreasing temperature. 425 Percent increase in cumulative osmotic stress for the $100 \mathrm{mM}$ trehalose and $300 \mathrm{mM}$ 426 trehalose curves relative to $0 \mathrm{mM}$ added trehalose. A magnified inset is provided to show 427 the largest difference occurs at the onset of freezing ranging from approximately $-3^{\circ} \mathrm{C}$ to $428-20^{\circ} \mathrm{C}$.

429 Fig. 6. Membrane integrity and growth pattern of cells after cryopreservation. A) 430 Membrane integrity measured immediately after thawing $\left({ }^{*}, p<0.01\right)$ and $\left.B\right)$ cell grow-out 431 after the LN2 storage for each CPA with sigmoidal fits to highlight growth patterns ( $n=4$, $432 \pm$ ISEM).

433 Fig.7. Recovery of cellular respiration over 3 days after cryopreservation. A) basal 434 respiration rate of cells, B) proton leak related respiration rates, C) FCCP uncoupled 435 maximum respiration, and D) non-mitochondrial oxygen consumption ( $n=3-6, \pm S E M)$.

436 Supplementary Figure I: Confocal Raman hyperspectral images of ice formation in 437 presence of $100 \mathrm{mM}$ and $300 \mathrm{mM}$ trehalose at $-40^{\circ} \mathrm{C}$ and $-80^{\circ} \mathrm{C}$. The images are generated 438 by integrating the ice peak $\left(\sim 3130 \mathrm{~cm}^{-1}\right)$ from the corresponding Raman spectra.

439 Supplementary Figure II: Numerical representation of crystal morphological properties 440 extracted from Raman hyperspectral images. Images were taken at $-40^{\circ} \mathrm{C}$ and $-80^{\circ} \mathrm{C}$ for 441 each of the two CPA solutions containing only trehalose $(100 \mathrm{mM}$, and $300 \mathrm{mM})$. 442 Hyperspectral images were analyzed with ImageJ to extract average number of ice 443 crystals (A) and average area per ice crystals (B) were found via ImageJ per sample 444 window $(n=3)$. 


\section{References:}

447 [1] T.J. Anchordoguy, A.S. Rudolph, J.F. Carpenter, and J.H. Crowe, Modes of interaction 448 449 of cryoprotectants with membrane phospholipids during freezing. Cryobiology 24 (1987) 324-331.

[2] A.S. Rudolph, and J.H. Crowe, Membrane stabilization during freezing: The role of two natural cryoprotectants, trehalose and proline. Cryobiology 22 (1985) 367-377.

452

[3] J.P. Rodrigues, F.H. Paraguassú-Braga, L. Carvalho, E. Abdelhay, L.F. Bouzas, and L.C. Porto, Evaluation of trehalose and sucrose as cryoprotectants for

[4] B. Stokich, Q. Osgood, D. Grimm, S. Moorthy, N. Chakraborty, and M.A. Menze, hematopoietic stem cells of umbilical cord blood. Cryobiology 56 (2008) 144-151. Cryopreservation of hepatocyte (HepG2) cell monolayers: Impact of trehalose. Cryobiology 69 (2014) 281-290.

[5] T.L. Bailey, M. Wang, J. Solocinski, B.P. Nathan, N. Chakraborty, and M.A. Menze, Protective effects of osmolytes in cryopreserving adherent neuroblastoma (Neuro2a) cells. Cryobiology 71 (2015) 472-480.

[6] T. Isobe, Y. Ikebata, T. Onitsuka, L.T.K. Do, Y. Sato, M. Taniguchi, and T. Otoi, Cryopreservation for bovine embryos in serum-free freezing medium containing silk protein sericin. Cryobiology 67 (2013) 184-187.

[7] J.D. Gantz, and R.E. Lee Jr, The limits of drought-induced rapid cold-hardening: Extremely brief, mild desiccation triggers enhanced freeze-tolerance in Eurosta 466 solidaginis larvae. Journal of Insect Physiology 73 (2015) 30-36. 
467 [8] J.H. Crowe, L.M. Crowe, W.F. Wolkers, A.E. Oliver, X. Ma, J.-H. Auh, M. Tang, S. Zhu, 468 J. Norris, and F. Tablin, Stabilization of Dry Mammalian Cells: Lessons from Nature. Integrative and Comparative Biology 45 (2005) 810-820.

470

[9] P. Alpert, The Limits and Frontiers of Desiccation-Tolerant Life. Integrative and 471 Comparative Biology 45 (2005) 685-695.

472 [10] S.S. Buchanan, S.A. Gross, J.P. Acker, M. Toner, J.F. Carpenter, and D.W. Pyatt, 473

[12] A. Eroglu, M.J. Russo, R. Bieganski, A. Fowler, S. Cheley, H. Bayley, and M. Toner, Intracellular trehalose improves the survival of cryopreserved mammalian cells. Nature biotechnology 18 (2000) 163-167.

[13] J.H. Crowe, Trehalose as a "chemical chaperone": fact and fantasy. Adv Exp Med Biol 594 (2007) 143-58.

[14] J.H. Crowe, L.M. Crowe, A.E. Oliver, N. Tsvetkova, W. Wolkers, and F. Tablin, The trehalose myth revisited: introduction to a symposium on stabilization of cells in the dry state. Cryobiology 43 (2001) 89-105.

[15] J.G. Baust, D. Gao, and J.M. Baust, Cryopreservation: An emerging paradigm change. Organogenesis 5 (2009) 90-96. 
[16] H.T. Meryman, R.J. Williams, and M.S. Douglas, Freezing injury from "solution effects" and its prevention by natural or artificial cryoprotection. Cryobiology 14 (1977) 287-302.

[17] G. Rapatz, and B. Luyet, Combined effects of freezing rates and of various protective agents on the preservation of human erythrocytes. Cryobiology 4 (1968) 215-22.

[18] P. Mazur, Life in the frozen state. Principles of Cryobiology. CRC Press, Boca Raton, FL, USA. Academic Press. Boca Raton (2004) 3-65.

[19] H. Takamatsu, and S. Zawlodzka, Contribution of extracellular ice formation and the solution effects to the freezing injury of PC-3 cells suspended in $\mathrm{NaCl}$ solutions. Cryobiology 53 (2006) 1-11.

[20] G.J. Puppels, F.F.M. de Mul, C. Otto, J. Greve, M. Robert-Nicoud, D.J. Arndt-Jovin, and T.M. Jovin, Studying single living cells and chromosomes by confocal Raman microspectroscopy. Nature 347 (1990) 301-303.

[21] K. Kneipp, H. Kneipp, I. Itzkan, R.R. Dasari, and M.S. Feld, Ultrasensitive chemical analysis by Raman spectroscopy. Chemical reviews 99 (1999) 2957-2976.

[22] J.W. Chan, D.S. Taylor, T. Zwerdling, S.M. Lane, K. Ihara, and T. Huser, MicroRaman spectroscopy detects individual neoplastic and normal hematopoietic cells. Biophysical journal 90 (2006) 648-656.

[23] Q. Du, R. Superfine, E. Freysz, and Y. Shen, Vibrational spectroscopy of water at the vapor/water interface. Physical Review Letters 70 (1993) 2313.

[24] J. Dong, J. Malsam, J.C. Bischof, A. Hubel, and A. Aksan, Spatial Distribution of the State of Water in Frozen Mammalian Cells. Biophysical Journal 99 (2010) 24532459. 
511 [25] W. Rall, P. Mazur, and J. McGrath, Depression of the ice-nucleation temperature of rapidly cooled mouse embryos by glycerol and dimethyl sulfoxide. Biophysical journal 41 (1983) 1.

[26] P. Mazur, Freezing of living cells: mechanisms and implications. American Journal of Physiology-Cell Physiology 247 (1984) C125-C142.

[27] X. Chen, W. Hua, Z. Huang, and H.C. Allen, Interfacial water structure associated with phospholipid membranes studied by phase-sensitive vibrational sum frequency generation spectroscopy. Journal of the American Chemical Society 132 (2010) 11336-11342.

[28] C. Branca, S. Magazu, G. Maisano, and P. Migliardo, Anomalous cryoprotective effectiveness of trehalose: Raman scattering evidences. The Journal of chemical physics 111 (1999) 281-287.

[29] M.C. Donnamaria, E.I. Howard, and J.R. Grigera, Interaction of water with a, atrehalose in solution: molecular dynamics simulation approach. Journal of the Chemical Society, Faraday Transactions 90 (1994) 2731-2735.

[30] A. Lerbret, P. Bordat, F. Affouard, M. Descamps, and F. Migliardo, How homogeneous are the trehalose, maltose, and sucrose water solutions? An insight from molecular dynamics simulations. The Journal of Physical Chemistry B 109 (2005) 11046-11057.

[31] J.L. Green, and C.A. Angell, Phase relations and vitrification in saccharide-water solutions and the trehalose anomaly. The Journal of Physical Chemistry 93 (1989) 2880-2882. 
[32] S.N. Timasheff, The Control of Protein Stability and Association by Weak Interactions with Water: How Do Solvents Affect These Processes? Annual Review of Biophysics and Biomolecular Structure 22 (1993) 67-97.

[33] T. Uchida, M. Nagayama, and K. Gohara, Trehalose solution viscosity at low temperatures measured by dynamic light scattering method: Trehalose depresses molecular transportation for ice crystal growth. Journal of Crystal Growth 311 (2009) 4747-4752.

[34] M.D. Abràmoff, P.J. Magalhães, and S.J. Ram, Image processing with ImageJ. Biophotonics International 11 (2004) 36-42.

[35] L.J. Belaid, and W. Mourou, Image segmentation: a watershed transformation algorithm. Image Analysis \& Stereology 28 (2009) 93-102.

[36] B.T. Storey, E.E. Noiles, and K.A. Thompson, Comparison of glycerol, other polyols, trehalose, and raffinose to provide a defined cryoprotectant medium for mouse sperm cryopreservation. Cryobiology 37 (1998) 46-58.

[37] F. Lang, G.L. Busch, M. Ritter, H. VÖLKL, S. Waldegger, E. Gulbins, and D. HÄUSSINGER, Functional significance of cell volume regulatory mechanisms. Physiological reviews 78 (1998) 247-306.

[38] D. Pegg, The history and principles of cryopreservation, Seminars in reproductive medicine, Copyright@ 2002 by Thieme Medical Publishers, Inc., 333 Seventh Avenue, New York, NY 10001, USA. Tel.:+ 1 (212) 584-4662, 2002, pp. 005-014.

[39] W. Rall, Factors affecting the survival of mouse embryos cryopreserved by vitrification. Cryobiology 24 (1987) 387-402. 
[40] G.M. Fahy, Simplified calculation of cell water content during freezing and thawing in nonideal solutions of cryoprotective agents and its possible application to the study of "solution effects" injury. Cryobiology 18 (1981) 473-482.

[41] J.O. Karlsson, E.G. Cravalho, and M. Toner, Intracellular ice formation: causes and consequences. Cryo-Letters 14 (1993) 323-334.

[42] A. Fowler, and M. Toner, Cryo-injury and biopreservation. Annals of the new york academy of sciences 1066 (2006) 119-135.

[43] M. Schmehl, I. Vazquez, and E. Graham, The effects of nonpenetrating cryoprotectants added to TEST-yolk-glycerol extender on the post-thaw motility of ram spermatozoa. Cryobiology 23 (1986) 512-517.

[44] J.L. Hanslick, K. Lau, K.K. Noguchi, J.W. Olney, C.F. Zorumski, S. Mennerick, and N.B. Farber, Dimethyl sulfoxide (DMSO) produces widespread apoptosis in the developing central nervous system. Neurobiology of disease 34 (2009) 1-10.

[45] R. Notman, M. Noro, B. O'Malley, and J. Anwar, Molecular basis for dimethylsulfoxide (DMSO) action on lipid membranes. Journal of the American Chemical Society 128 (2006) 13982-13983.

[46] M.-A. de Ménorval, L.M. Mir, M.L. Fernández, and R. Reigada, Effects of dimethyl sulfoxide in cholesterol-containing lipid membranes: a comparative study of experiments in silico and with cells. PloS one 7 (2012) e41733.

[47] G.M. Fahy, T.H. Lilley, H. Linsdell, M.S.J. Douglas, and H.T. Meryman, Cryoprotectant toxicity and cryoprotectant toxicity reduction: in search of molecular mechanisms. Cryobiology 27 (1990) 247-268. 
[48] S.K. Singh, P. Kolhe, A.P. Mehta, S.C. Chico, A.L. Lary, and M. Huang, Frozen state storage instability of a monoclonal antibody: Aggregation as a consequence of trehalose crystallization and protein unfolding. Pharmaceutical research 28 (2011) 873-885.

[49] Y. Momose, R. Matsumoto, A. Maruyama, and M. Yamaoka, Comparative analysis of transcriptional responses to the cryoprotectants, dimethyl sulfoxide and trehalose, which confer tolerance to freeze-thaw stress in Saccharomyces cerevisiae. Cryobiology 60 (2010) 245-261.

[50] J.P.R. Motta, F.H. Paraguassú-Braga, L.F. Bouzas, and L.C. Porto, Evaluation of intracellular and extracellular trehalose as a cryoprotectant of stem cells obtained from umbilical cord blood. Cryobiology 68 (2014) 343-348.

[51] L.M. Crowe, D.S. Reid, and J.H. Crowe, Is trehalose special for preserving dry biomaterials? Biophysical journal 71 (1996) 2087.

[52] E. Katenz, F.W.R. Vondran, R. Schwartlander, G. Pless, X. Gong, X. Cheng, P. Neuhaus, and I.M. Sauer, Cryopreservation of primary human hepatocytes: the benefit of trehalose as an additional cryoprotective agent. Liver transplantation 13 (2007) 38-45.

[53] G.M. Beattie, J.H. Crowe, A.D. Lopez, V. Cirulli, C. Ricordi, and A. Hayek, Trehalose: a cryoprotectant that enhances recovery and preserves function of human pancreatic islets after long-term storage. Diabetes 46 (1997) 519-523.

[54] J.H. Crowe, J.S. Clegg, and L.M. Crowe, Anhydrobiosis: the water replacement hypothesis, The Properties of Water in Foods ISOPOW 6, Springer, 1998, pp. 440455. 
600 [55] C. Erkut, S. Penkov, K. Fahmy, and T.V. Kurzchalia, How worms survive desiccation: Trehalose pro water, Worm, Taylor \& Francis, 2012, pp. 61-65.

602 [56] K. Muldrew, and L.E. McGann, The osmotic rupture hypothesis of intracellular 603 freezing injury. Biophysical Journal 66 (1994) 532.

604 [57] J. Huebinger, H.-M. Han, O. Hofnagel, I.R. Vetter, P.I. Bastiaens, and M. 605 606 607 608 609 610 Grabenbauer, Direct Measurement of Water States in Cryopreserved Cells Reveals Tolerance toward Ice Crystallization. Biophysical journal 110 (2016) 840849.

[58] K. Brockbank, F. Lightfoot, Y.C. Song, and M. Taylor, Interstitial ice formation in cryopreserved homografts: a possible cause of tissue deterioration and calcification in vivo. The Journal of heart valve disease 9 (2000) 200-206.

[59] B. Minceva-Sukarova, W. Sherman, and G. Wilkinson, The Raman spectra of ice (Ih, II, III, V, VI and IX) as functions of pressure and temperature. Journal of Physics C: Solid State Physics 17 (1984) 5833. 


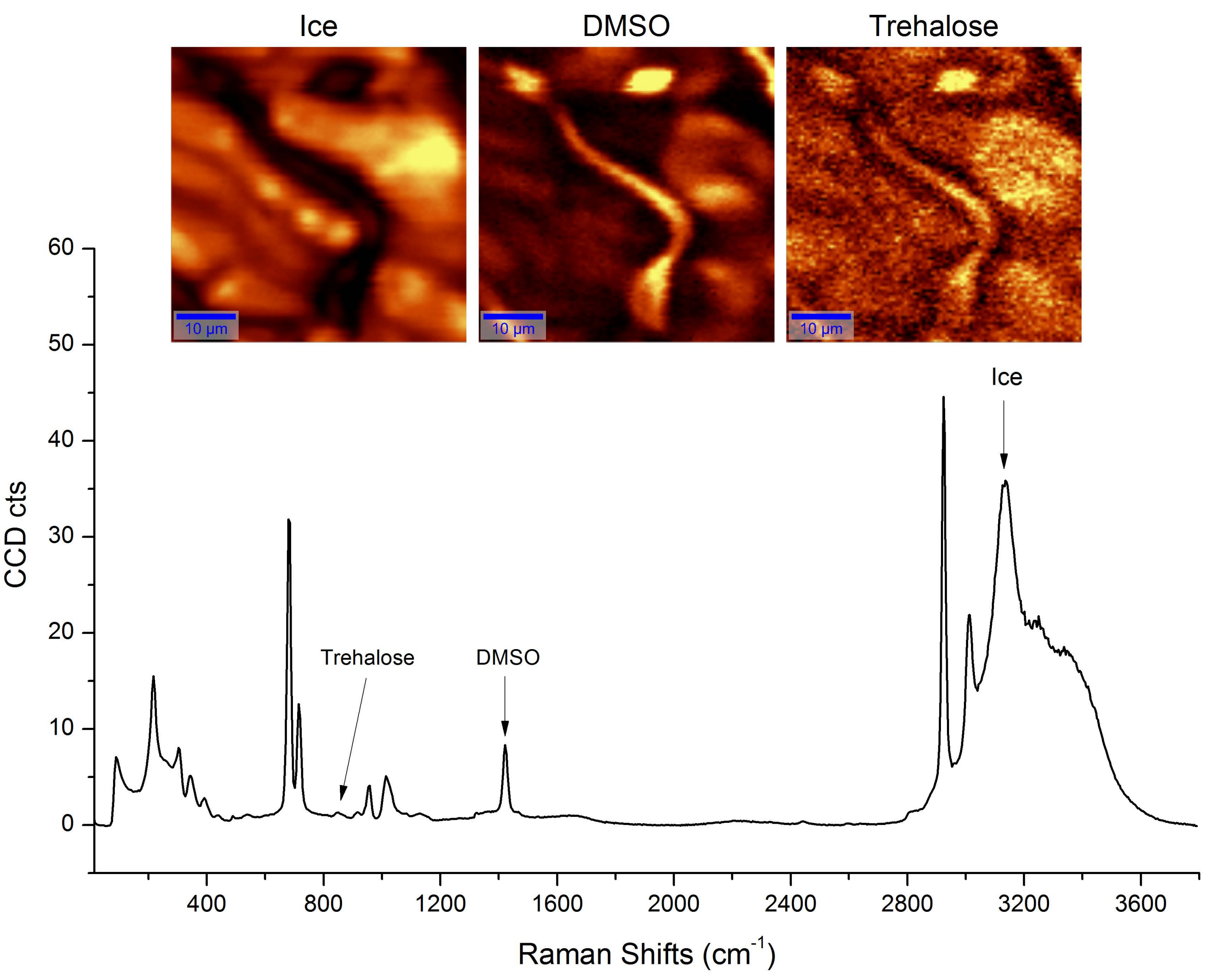



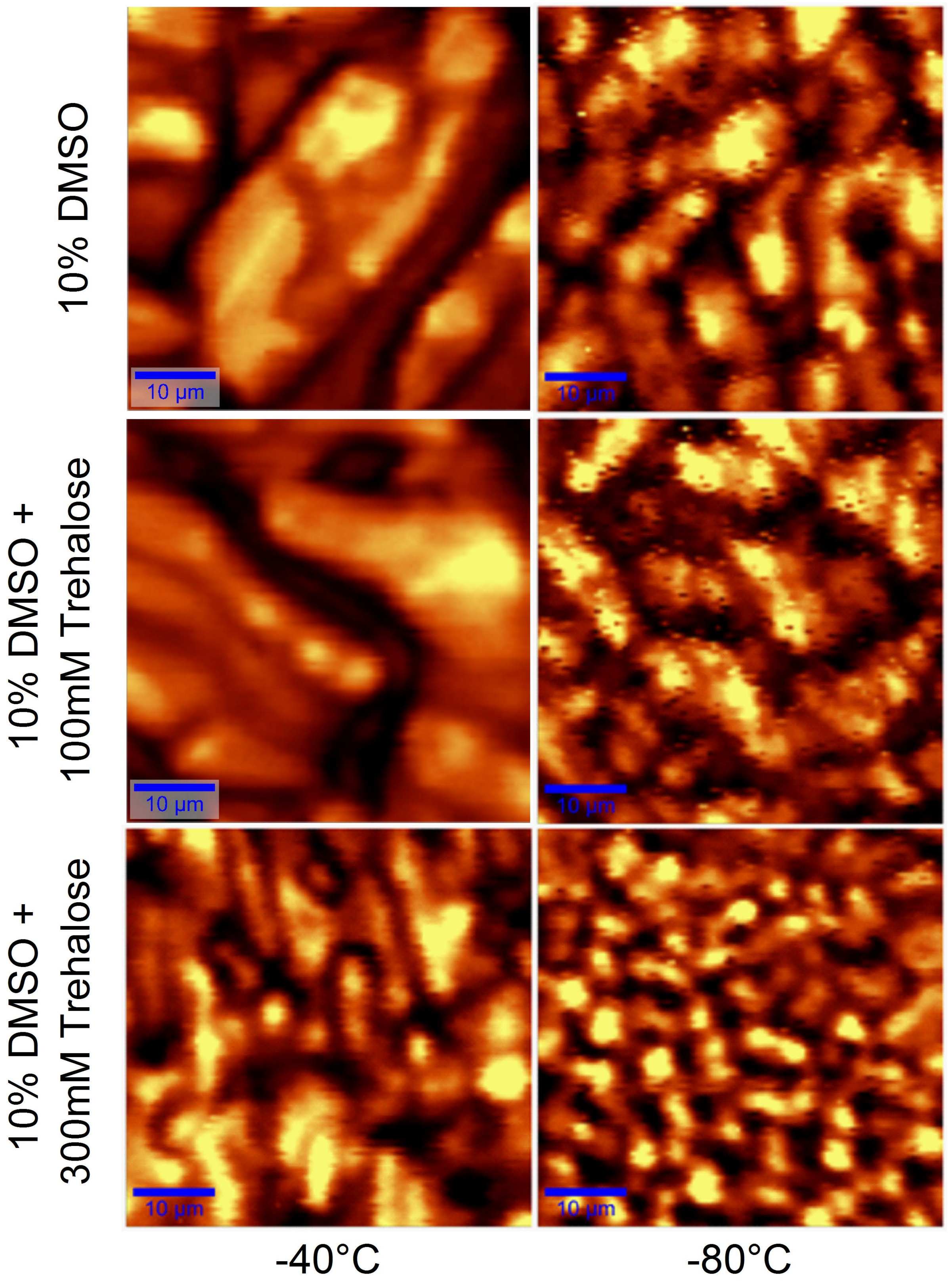

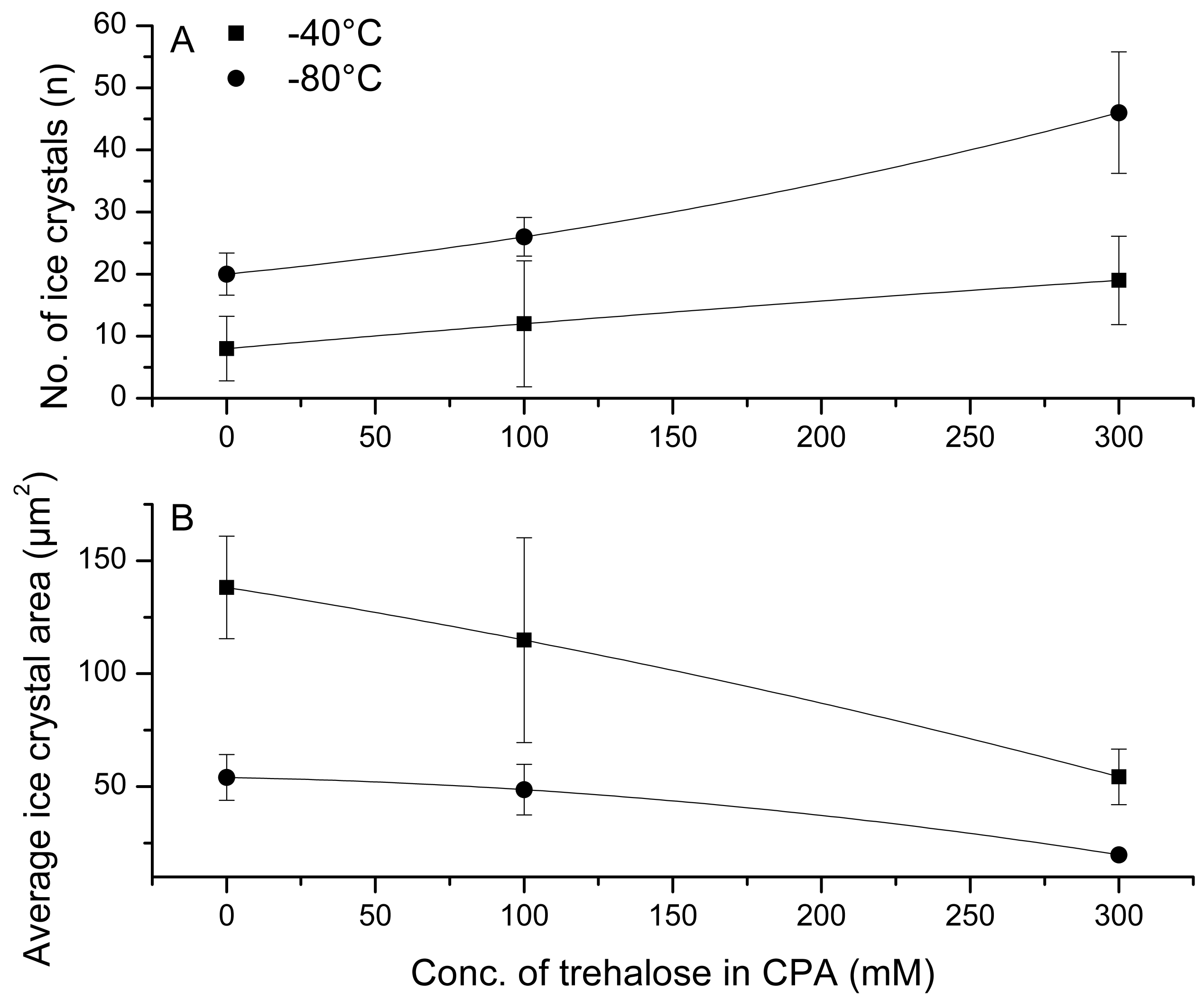


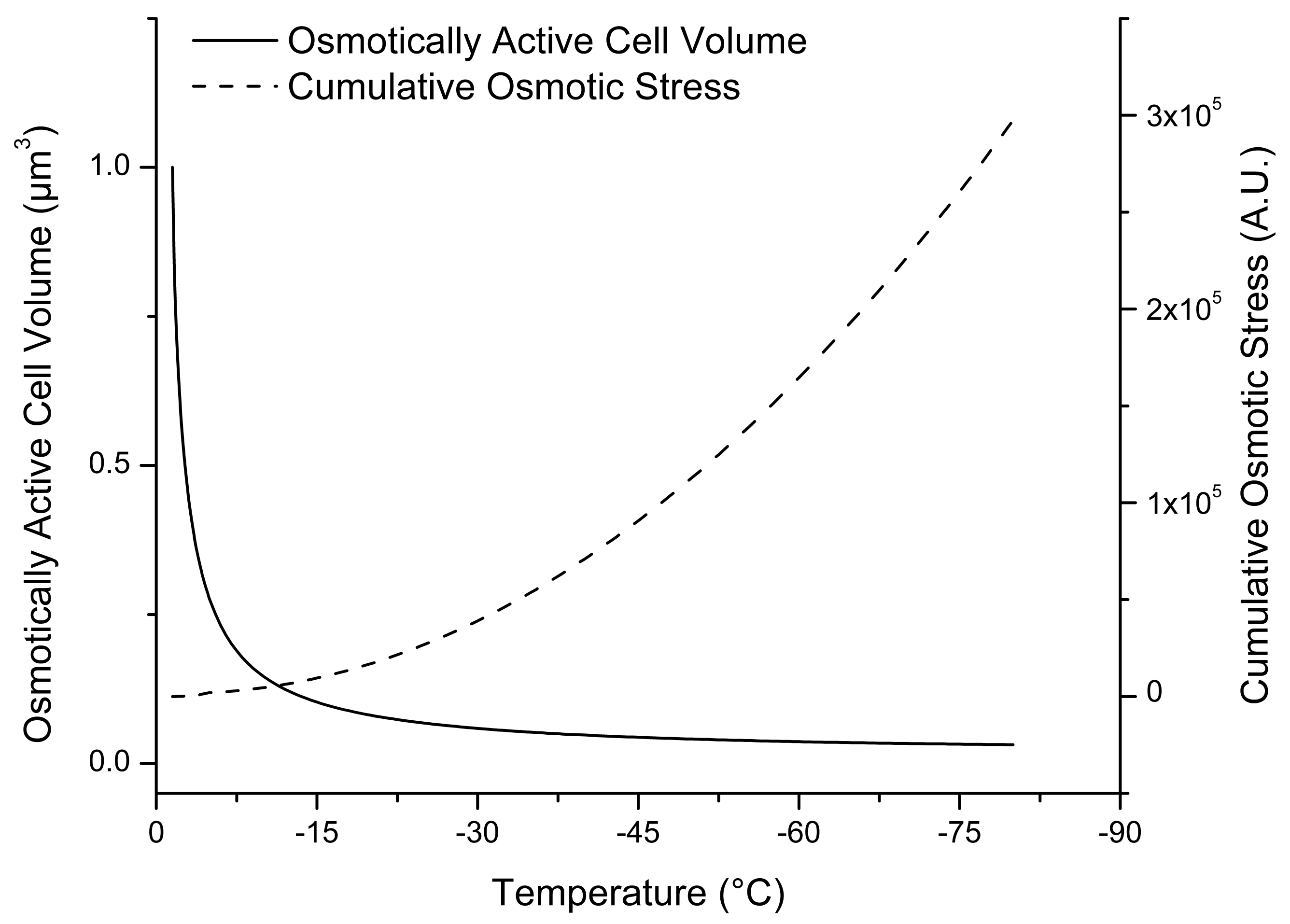




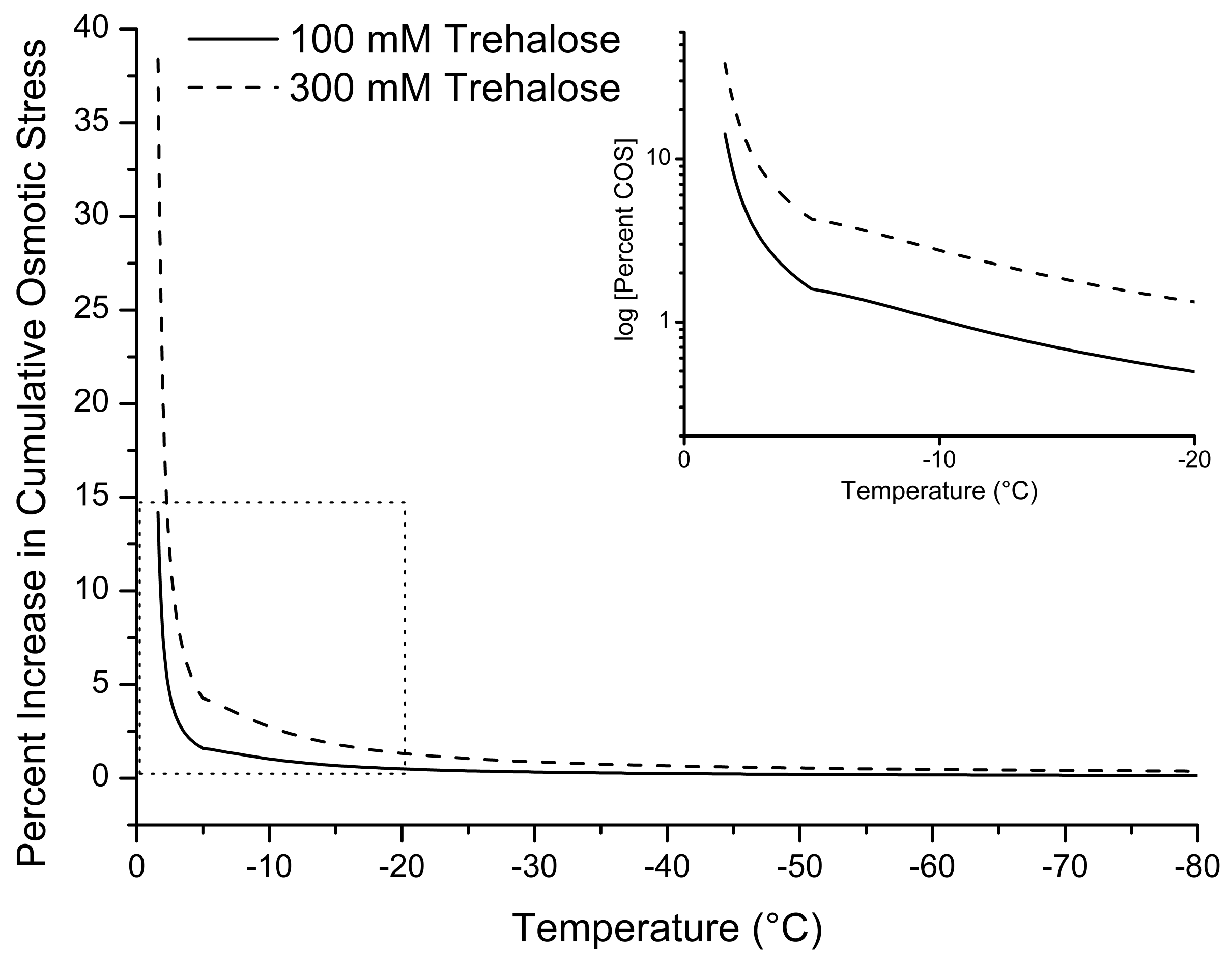



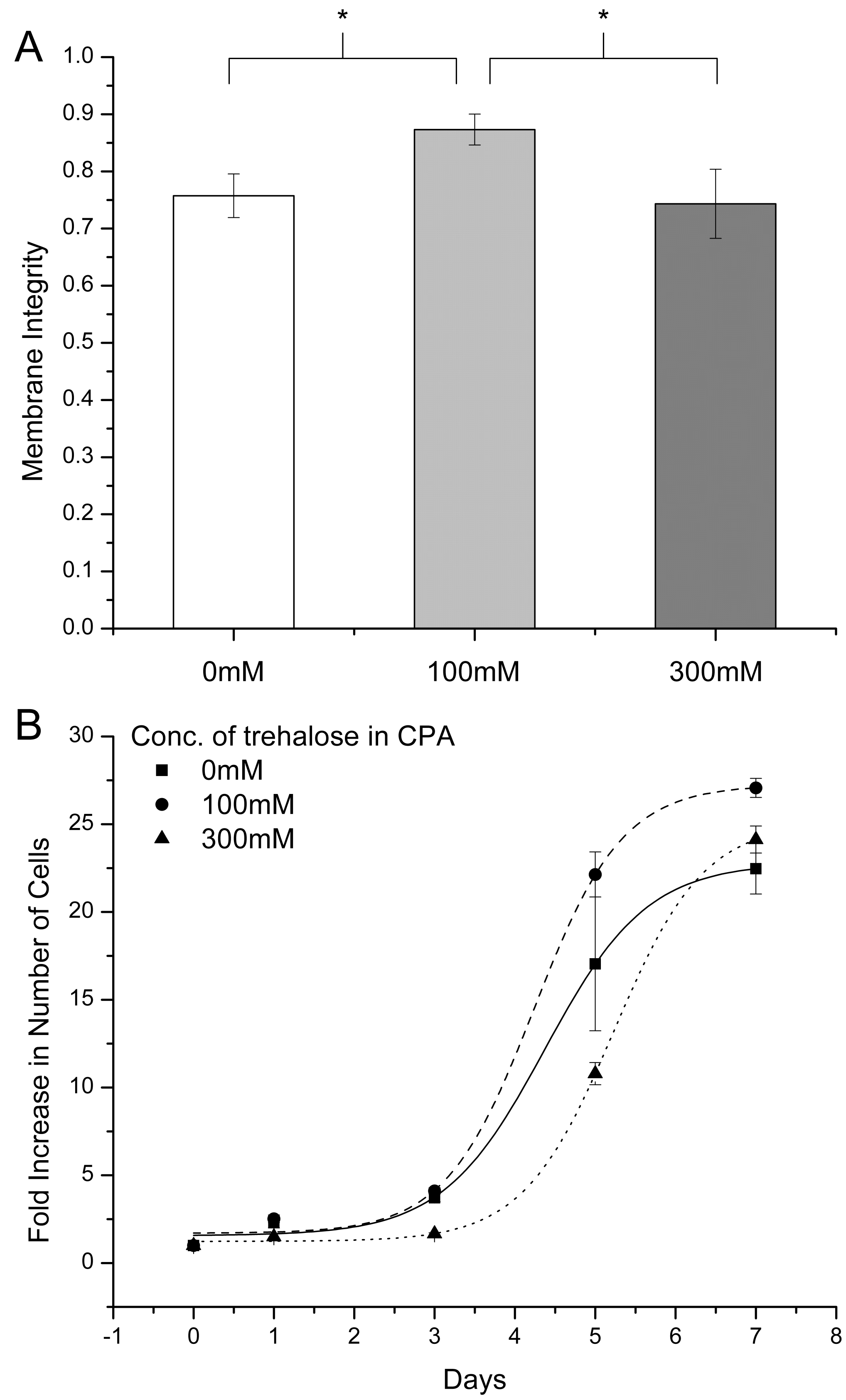
A

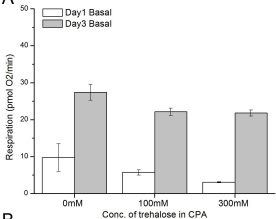

B

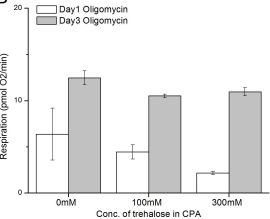

C

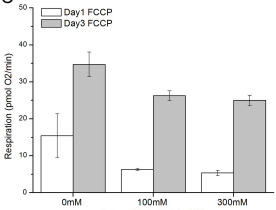

D

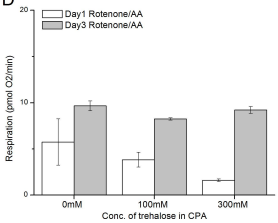

